\title{
OPPORTUNITIES AND CHALLENGES OF NANOTECHNOLOGY FOR COMPUTER SCIENCE
}

\author{
Dr.S.Angel Latha Mary ${ }^{1 *}$, E.Rama Kalaivani ${ }^{2}$, Ms.K.Uma-Maheswari ${ }^{3}$ \\ *l Professor/ITKarpagam College of Engineering, Coimbatore, India, \\ ${ }^{2,3}$ Assistant Professor/CSEKarpagam College of EngineeringCoimbatore, India \\ *1xavierangellatha@gmail.com, ${ }^{2}$ ramakalaivani.e@gmail.com \\ 3umamaheswarikit@gmail.com
}

\section{*Corresponding Author: -}

Email ID - xavierangellatha@ gmail.com

\begin{abstract}
: -
It was in 1959, Prof. Richard Feynman, a future Nobel prize winner delivered a visionary talk entitled, "There's plenty of Room at the bottom", visualizing the possibility of manipulating single atoms and molecules to produce stunning discoveries. Though at that time, Feynman's talk looked very theoretical, now we are on the brink of actualizing that dream. This approach of atomic scale fabrication was termed by Feyman as 'bottom up approach', as opposed to the conventional method of 'top down approach' for material fabrication. According to the bottom up approach, building products with atomic precision will offer large potentials in all fields of human life. The term 'Nanotechnology' was introduced by Drexeler to describe Feynman's grand vision. And it has inspired many researches to carry further the application of Nanotechnology to various scientific and application fields. Nanotechnology has been defined as the fabrication of devices with atomic or molecular scale precision. Devices with minimum features and sizes less than 100 nanometer $(\mathrm{nm})$ are considered to the products of nanotechnology. As Nanotechnology enables us to organize matter at the atomic scale there are numerous fields of study that stand to gain.According to computer scientists, if the computer industry does not make radical changes to the fundamentals of computer technology, computing power will reach its physical limits. Moore's Law states that the number of transistors that can be fitted on a chip doubles every eighteen months. However, current silicon technologies are approaching the limits imposed by quantum mechanics.
\end{abstract}

Keywords: - Nanotechnology, Quantum Computing, Quantum dots. 


\section{FUTURE PROMISES AND FEARS OF NANOTECHNOLOGY}

Nanotechnology is an inter-disciplinary field of study that involves interests from the fields of physics, chemistry, biology, medicine, computer science etc. Our focus here is to see the development and research that can aid and propel inventions specifically in the field of computer science. Every field of science, medicine, engineering, commerce and day to day applications stand to gain a lot from nanotechnology. There are also lot of fears regarding the future impact of nanotechnology on human life and environment. Approached with pessimism, Nanotechnology appears too dangerous. But looking positively we can see that it offers promises that cannot be simply thrown away. The promises are too good that our fear should not keep us away from exploring these possibilities. However, what is needed is guidance and ethical norms to keep in check destructive use of such a technology. We will now see in particular the importance of Nanotechnology to the field of computer science.

\section{NANOTECHNOLOGY FOR COMPUTER SCIENCE}

Nanotechnology seems to hold unlimited possibilities for the field of computer science. Let us briefly run through the basic achievements and possible routes that are opening up before the computing world. One of the innovations achieved in the field of Nanotechnology is the fabrication of Carbon Nanotubes discovered by S Lijima of NEC in 1991. Carbon Nanotubes have since found various applications. Researchers have successfully created electronic components including transistors, diodes, relays and logic gates from carbon Nanotubes.

Another new material is the Quantum Dots. They are crystals that emit only one wavelength of light when their electrons are excited. The electrical, magnetic and optical properties of these dots can be controlled by regulating their chemical reaction. The emission of light by quantum dots can be used as quantum bits to from the basic information interchange. We will see this in detail later.This will open a new area of growth in the computer field. As Nanotechnology-fabrication at various levels is progressing, researchers have managed to create electronic components from carbon Nanotubes. Research is now on to achieve interconnection between such components using nano-based materials. This will surely lead to the actualization of a silicon based nano-computer.

There are various areas of computers that stand to gain from the innovations of Nanotechnology. The data storage field is an area where number of different technologies are at work. Memories that in the form of ultradense, very large, nonvolatile random access memories (RAM), look pretty certain within the next few years. There are number of technologies at lower levels being pursued for memory applications. There are extremely diverse set of approaches like using arrays of tiny cantilevers with very sharp points to write magnetically, optically or even to make indentations in a polymer; there are also researches into making magnetic RAMs relying on quantum mechanical effects based on the spin of an electron; there are various types of molecular memory being pursued; and one promising approach is the one using carbon nano tubes. A few of these technologies promise memories so big that your personal computer may no longer need a hard drive. The production of displays with low energy consumption could be accomplished using carbon nano tubes. These can be electrically conductive and due to their small diameter of several nanometers, they can be used as field emitters with extremely high efficiency for Field Emission Displays (FED). The principle of operation will very much resemble that of the cathode ray tube, but on a much smaller length scale.

\section{NANO-RELATED COMPUTER \\ RESEARCHES}

Many established companies are making significant breakthroughs in the field of nanotechnology. Listed below are few of these:

- IBM: The IBM researchers have already successfully created carbon nano tube transistors that substantially outperform advanced silicon devices.

- Hewlett-Packard: The researchers at HP have patented a potential breakthrough in their quest to develop computer circuits made merely of individual molecules. HP hopes to refine the process to create microchips as powerful as the next generation of siliconbased chips, but thousand times smaller and less expensive.

- Intel recently announced a breakthrough in the design of chops that will enable the development of cheaper and faster microprocessors based on nao-level technology with more than 1 billion transistors compared to 125 million in Intel's latest Pentium IV chip.

- Applied Materials is building 'nano chips', tiny computer chips to power mobile devices. They expect that high speed wireless networks will drive demand for such chips.

- Nanogram in Fremont is an example of a leading company developing the core process technology enabling the manufacture of nanoscale optical, electronic and energy storage application.

\section{NANO-COMPUTERS}

We can we can look at two different approaches to Nano computers. They are Quantum Computing and Molecular computing. These two methods are very interrelated and they promise to replace semiconductors in our conventional chips. Quantum computing aims to process information at quantum level. At this level, unlike traditional computers, information is presented as quantum bits or Qubits, which can be in a state of 0,1 or super position(simultaneously 0 and 1). As quantum bits can hold multiple states simultaneously, millions of computations can be done at a given time. However, as we are dealing with matter at quantum level, there will be obstacles of quantum mechanics. Research is on to overcome this. 
Another approach is Molecular Computing. Here we seek to read, write and process information within a single molecule. One molecule that has proved promising for this computation is Deoxyribonucleic Acid (DNA) that carries genetic information. DNA is a long polymer made of four different nucleotides that can be represented by the letter A, T, C and G. The sequence of these nucleotides within DNA provides information for using DNA and its various proteins to carry out computations that are difficult. The advantage of molecular computing using DNA is that it can store enormous information than any memory chip and so can be used to perform parallel computing. Hence, the convergence of bioscience and information technology will extend well beyond their own individual scope, leading to a combined field of research. And the promises will be much rewarding. In the sections that follow we will see, a detailed write up on Quantum Computer.

\section{QUANTUM COMPUTING}

The high speed modern computer sitting in front of us is fundamentally no different from its 30 ton ancestors that were equipped with some 18000 vacuum tubes and miles of wiring. Though they have become considerably fast and compact in size, the technology has not been any different from that of the first computer proposed by Charles Babbage and the eventual creation by Konard Zuse in 1941. In a classic digital computer, as used by us today, a bit (0 or 1) is the fundamental unit of information. Each of these binary bits is physically realized through a macroscopic physical system, such as the magnetization on a hard disk platter or the charge on a capacitor. Hence a document having n-characters stored on a disk of the computer comprises of $8 \mathrm{n}$ bits of $1 \mathrm{~s}$ and 0 s. This classical approach is soon going to hit at a dead end. So we need to look at a new technology that will carry us forward. And here lies the opportunity to harness a new technology from quantum mechanics to realize a new mode of information processing.

In 1982, the Nobel prize-winning physicist Richard Feynman, thought of the idea of a 'Quantum Computer', a computer that uses the effects of quantum mechanics to its advantage. For long time, this idea of a quantum computer was mostly of theoretical interest only, but recent developments have brought the idea to everybody's focus.

In a classical model of a computer, the most fundamental building block can only exist in one of two distinct states of a 0 or a 1. But at quantum level the fundamental unit of information is a qubit. And it is no more binary in nature, but more quaternary. This qubit property arises as a direct consequence of its adherence to the laws of quantum mechanics. A qubit can exist not only in a state corresponding to the logical state of 0 or 1 as in a classical bit, but also in states corresponding to a blend or superposition of these classical states. In other words, a qubit can exist as a 0 , a 1 , or simultaneously as both 0 and 1 . However, this may look counterintuitive as everyday events are governed by classical physics. When a qubit is in this coherent superposition of both the states, it can be thought of as being in two universes. And any operation on such a qubit effectively acts on both the values at the same time. The advantageous point being that, by performing a single operation on a qubit, we have operated on two values. Significantly, for a two-qubit system would have four operations simultaneously. Hence, we see that increasing the number of qubits exponentially increases the 'quantum parallelism'.

\section{BUILDING A QUANTUM COMPUTER}

A quantum computer is not like a classical computer in design as it is not built from transistors and diodes. To build a quantum computer, we need a technology that enables 'qubits' to exist as coherent superposition of 0 and 1 state. The best method of achieving this is still unknown, but there are however, few methods being experimented with and some are proving success. Listed below are two such approaches:

\section{QUANTUM DOTS}

This is an example for the implementation of the qubit. It is basically a single electron trapped inside a cage of atoms. Exposing the dot to a pulse of Laser light of precise wavelength and duration, the electron is raised to an excited state; and a second burst of laser light causes it to fall back to its ground state. The ground and the excited states of the electron can be thought of as the 0 and 1 state of the qubit. And logically the laser light can be regarded as a controlled NOT function as it changes the qubit from 0 to 1 and visa versa.

If the pulse of the applied laser beam is only half the duration, then the electron is placed in a superposition of both ground and excited states simultaneously. This can be considered equivalent to the coherent state of the qubit. We can model more complex logic functions using the quantum dots arranged in pairs. But unfortunately there a number of practical problems that come on the way:

- The electron remains in its excited state only for about a microsecond before it falls back to the ground state and the required duration of each laser pulse is around 1 nano second. Hence there is a limit to the number of computational steps that can be performed before the information is lost.

- Constructing a quantum dots is a very difficult process because they are very small. A single dot measures just 1 nanometer. Further, the technology required to build a computer from these quantum dots does not still exist.

- In order to avoid cramming thousands of laser beams into a tiny space, quantum dots could be manufactured such that each responds to different frequencies of light. So a laser that could reliably retune itself would thus selectively target different groups of quantum dots with different frequencies of light. But this however is only at theoretical level, no technology exists as yet. 


\section{QUANTUM LIQUIDS}

The latest development in the area of quantum computing takes a radically new approach. It drops the assumption that the quantum medium has to be tiny and isolated from its surroundings. Instead this approach uses a sea of molecules to store the information. When the molecules are held in a magnetic field, each nucleus within the molecule spins in a certain direction, which can be used to describe its state; an upward spin would mean a1 and spinning down would mean a 0. Nuclear Magnetic Resonance (NMR) techniques can be used to detect these spin states and specific radio waves can flip the nuclei from spinning up (a state of 1 ) to spinning down (0) and vice-versa.

In the above case, the quantum computer is the molecule itself and the qubit is its nuclei within the molecule. This technique however does not use a single molecule but a 'mug' of liquid molecules. The advantage of this is that even if the molecules of the liquid bump into each other, the spin states of the nuclei within the molecule do not change. Even here, decoherence is still a problem, but the time before the de-coherence sets in, is much longer than other techniques. And the researchers believe that a few logical operations should be possible before the qubits begin to de-cohere. Dr. Gershenfield from the Massachusetts Institute of Technology is one of the pioneers of the liquid computing technique. His research team has already been able to add one and one together, that clearly shows that this technique is way beyond any other in trial. The key to being able to perform more complex tasks is to have more qubits but this requires more complex molecules with a greater number of nuclei. The caffeine molecule is a good choice for this. From what is achieved, the advancement to 10 qubit systems is apparently straightforward. And according to Dr. Gershenfield, such a system should be possible very soon.

\section{APPLICATIONS OF QUANTUM COMPUTERS}

We must note that a quantum computer will not necessarily outperform a classical computer at computational tasks, unless the program algorithms run on them are tailored to do so. In order for a quantum computer to show its superiority, it needs to use algorithms that exploit its real power of quantum parallelism. Such algorithms are difficult to formulate. To date, we have only two such significant algorithms, they are: Shor's algorithm and Grover's algorithm. By using these algorithms a quantum computer will be able to outperform its classical counter part by a huge margin. Shor's algorithm allows quick factorizing of large numbers. With algorithms that are in use today, a classical computer would take 10 million billion years to factor a 1000 digit number, where as a quantum computer would take around 20 minutes.

Grover's algorithm allows searching of an unsorted database having $\mathrm{N}$ entries, using the quantum parallelism. In his approach it is possible to do the search in root $\mathrm{N}$ searches. Here, using the quantum parallelism the database is effectively distributed over a multitude of universes and this allows a single search to locate the required entry.

\section{SIMULATIONS OF QUANTUM MECHANICAL SYSTEMS}

It was Feynman in 1982, who conjectured that quantum computers would be able to simulate quantum mechanical systems with greater degree of accuracy than classical computers. On a classical computer, the dynamics of quantum systems can only be simulated using approximations, but a quantum computer can be programmed to simulate such a system. As quantum computers imitate the characteristics of the system in hand, we can for example allow the 'Hubbard Model' (that describes the movement of electrons within a crystal) to be simulated on such a machine.

\section{QUANTUM COMMUNICATIONS}

The researches carried out in quantum computing have brought out another interesting spin off field of communication. Here, by using the properties of quantum mechanical effects, the area aims to provide secure communication mechanisms. Quantum communication makes use of the fact that the information can be encoded as the polarization of photons (i.e. the orientation of a photon's oscillation). Here the orientation in one direction (Rectilinear) can be thought of as 0 and in another (Diagonal) as a 1. In order to decode the data at the receiver's end, the photons have to be measured using the correct polarization, as the one used to transmit the information. Using this method information can be sent in such way as to make it impossible for an eavesdropper to listen the message undetected. The British Telecom is working on this area and the findings are giving quantum communications a promising future.

\section{ARTIFICIAL INTELLIGENCE}

The findings of quantum computation have some interesting implications in the world of artificial intelligence. The argument of artificial intelligence has always been, questioning the capacity of a machine to perform those that are done consciously by the human mind. The theory of quantum computing allows us to look at the question of consciousness from a new angle. Every physical object now can be looked upon as quantum computer, and awaking conscious computing in these objects is only simulating their natural physical process. And many feel that quantum computing is the key to bringing intelligence into computing. This however is still at the theoretical level.

\section{OBSTACLES AND PITFALLS OF QUANTUM COMPUTING}

The field of quantum computing has made numerous promises since its conception and researchers have managed building of two and three qubit quantum computers able to do simple arithmetic and data sorting operations. But, a few potentially large obstacles still remain that prevent us from building one that can rival today's modern digital computers. Among these difficulties, error correction, decoherence and hardware architecture are the most formidable ones. We see each in detail with their technical promises. 
Any essential piece of software for computing technology should have a way to correct errors. Machines make mistakes. Classical computers are designed to catch such errors through redundancy. They perform each elementary computation several times and then accept the most frequent answer as correct. But this approach will not work on a quantum machine, precisely as any observation before the computation will alter the effect Surprisingly, however, quantum error correction is possible. In 1995 the mathematician Peter W. Shor and the physicist Andrew M. Steane of the University of Oxford independently devised a scheme that deftly tiptoes through the quantum minefield. The scheme detects error in a way that reveals nothing about the ongoing computation (and thus does not ruin the superposition). Then it fixes the error by means of another quantum computation, which also keeps the quantum system intact.

The very thing that makes quantum computing so powerful also makes it very fragile and difficult to control. For example consider a qubit that is in the coherent state. As soon as it interacts with the environment, it will decohere and fall into one of the two classical states. This is the problem of decoherence. It is a stumbling block for quantum computers as the potential power of such systems depend solely on the quantum parallelism, brought about by the coherent state. This problem is compounded by the fact that even looking at a qubit can cause it to decohere, making the process of obtaining a solution from the quantum computer difficult. To counter this problem, quantum researchers utilize another aspect of quantum mechanics known as entanglement. To make a practical quantum computer, scientists have to devise ways of making measurements indirectly to preserve the system's integrity. Entanglement provides a potential answer. In quantum physics, if you apply an outside force to two atoms, it can cause them to become entangled and the second atom can take on the properties of the first atom. So if left alone, an atom will spin in all directions. The instant it is disturbed it chooses one spin, or one value; and at the same time, the second entangled atom will choose an opposite spin, or value. This allows scientists to know the value of the qubits without actually looking at them.

In 1998 researches at Los Alamos National Laboratory and MIT led by Raymond Laflamme managed to spread a single qubit across three nuclear spins in each molecule of a liquid solution of alanine(trichloroethylene) molecules. They achieved this using the techniques of Nuclear Magnetic Resonance (NMR). This experiment is important, because spreading out the information actually made it harder to corrupt the data. So as a result of several significant experiments, nuclear magnetic resonance has become the most popular component in quantum hardware architecture.

\section{CONCLUSION}

Nanotechnology has arrived and we are yet to realize its full potential. Our computers of today are good enough, but no revolutionary break through has taken place in the field since the invention of transistors. As the world of nanotechnology hold great possibilities, computer scientists must focus to build systems that consist of large number of nanolevel particles that automatically form into a large scale integrated system capable of high end and efficient computing.

\section{REFERENCES}

[1].Boonserm Kaewkamnerdpong and Peter J.Bentley, Computer Science for Nanotechnology: Needs and Opportunities. Department of Computer Science, University College London, UK.

[2].http://www.nanoword.net/pages/intro.htm

[3].http://www.nanotechnow.com/nano_intro.htm

[4].http://www.zdnetindia.com/news/hardware/st oreis/172435.html

[5].http://www.hindu.com/seta/2005/08/04/index .html

[6].http://www.presidentofindia.gov.in/presentati on/splangnewPDF\%20Format1030.pdf

[7].http://outlookindia.com/wired_files

[8].http://in.news.yahoo.com

[9].http://www.rediff/money/inter.html

[10]. http://in.answers.yahoo.com/dir

[11]. http://economictimes.indiatimes.com/infotec h/software/articlelist/13357555.cms

[12]. http://www.cs.caltech.edu/ westside/quantu m-intro.html

[13]. http://www.doc.ic.ac.uk/ nd/surprise_97/jou rnal/vol4/spb3/\#1. Introduction

[14]. http://cryptome.org/qc-grover.htm

[15]. http://www.research.ibm.com/quantuminfo/

[16]. http://wwwsers.cs.york.ac.uk/schmuel/comp/comp.html 